\title{
Development of ANN Model for Prediction of Coating Thickness in Hot Dip Galvanizing Process
}

\author{
Sanjeev Kumar Shukla1*, Anup Kumar Sadhukhan², Parthapratim Gupta ${ }^{2}$ \\ 1 Steel Products Group, Research and Development Centre for Iron Steel, Steel Authority of India, Ranchi-834002, \\ India. \\ ${ }^{2}$ Chemical Engineering Department, National Institute of Technology, Durgapur-713209, India. \\ * Corresponding author: Tel: +91-8986889279; email: skshukla@sail-rdcis.com \\ Manuscript submitted October 21, 2016; accepted February 4, 2017. \\ doi: 10.17706/ijmse.2017.5.2.60-68
}

\begin{abstract}
Hot dip galvanizing of steel strip is carried out in Continuous Galvanizing Lines (CGL) in order to achieve consistency in thickness \& quality of Zn-coating. The process is a complex one and is always in dynamic equilibrium depending on various process parameters. Around 20 process parameters, grouped into major sections like steel strip characteristics, pre-treatment, galvanizing process conditions, bath conditions, post treatment operations, are involved in the process. Zn-coating thickness is a nonlinear and coupled function of most of the above process input parameters. In the present work Artificial Neural Network (ANN) has been used to develop an off-line model to predict the coating thickness. Six factors that were identified through sensitivity analysis based on Taguchi's Orthogonal Array Technique were used as independent variables in the ANN model. A Hot Dip Process Simulator (HDPS) was used to generate the input-output data with close control of input process parameters. The data set generated for training purpose has the coating thickness variation between 12 to $32 \mu \mathrm{m}$. The model, developed with total data-sets (234), has the prediction error of $10 \%$. However, the maximum data-sets (183) lie in the range of 20-30 $\mu \mathrm{m}$ and model developed by taking these datasets in this range has the prediction error of 7\%. In both the cases a 6-(9-6-3)-1 network structure was used \& $3 / 4$ of data-sets were used for training \& testing \& $1 / 4$ for validation.
\end{abstract}

Keywords: Galvanizing process, coating thickness, artificial neural network, predictive model.

\section{Introduction}

Hot dip galvanizing of steel strip is generally carried out in Continuous Galvanizing Lines (CGL) to achieve consistency in product quality and high line productivity. Galvanizing bath contains mostly pure zinc. Certain metallic elements like aluminium ( $\mathrm{Al}$ ), lead $(\mathrm{Pb})$, Antimony $(\mathrm{Sb})$ etc. are specially added to the bath to enhance coating adhesion, appearance (lustre and spangle finish) and processing efficiency. Most galvanizing lines add $0.18-0.22 \%$ of $\mathrm{Al}$ to zinc bath. The addition of $\mathrm{Al}$ is primarily to promote the formation of $\mathrm{Fe}_{2} \mathrm{Al}_{5}$ layer or inhibition layer. Hot dip galvanizing is a complex process under constant change and dynamic equilibrium. Around 20 parameters, grouped into major sections like steel strip characteristics, pre-treatment, galvanizing process conditions, bath conditions, post treatment operations play important roles in the process. Zn-coating thickness bears nonlinear and coupled relationship with most of the above process input parameters. The 
importance of controlling the coating thickness \& its uniformity for quality \& cost of galvanized sheet has been well established [1]-[6].

During the last few decades, model based process control in CGL has been reported by many researchers [7][15]. Some of the models have been developed through experimentation in simulator. As the conventional Regression Analysis (RA) is not suitable to assess the effect of such non-linear \& interacting process input-output relationship, Artificial Neural Network (ANN) was chosen as the tool to develop a Predictive Mathematical Model. The concepts \& application of Artificial Neural Network (ANN) based process control for galvanizing and other manufacturing processes have been described elsewhere [16]-[24].

Presently, on-line model based control of Air-knife wiping system for control of coating thickness \& its uniformity uses any three or four of the following process variables as inputs:

i) Strip to knife distance ( $\mathrm{mm})$

ii) Strip speed (m/min)

iii) Wiping gas pressure at nozzle (mbar)

iv) Nozzle opening ( $\mathrm{mm})$

This article describes the process and outcome of the development of an off-line ANN model to predict the coating thickness based on six process input parameters/factors. Hot Dip Process Simulator (HDPS), a laboratory-scale experimental set-up to study the actual galvanizing process, was used for experimentations and data generation. An ANN model was developed, trained and validated by the experimental data.

\section{Mathematical Model}

\subsection{Modeling for Galvanizing Process}

The Zn-coating thickness depends on the chemical kinetics associated with transport limitations. Linares proposed the first mathematical model, which depicted the diffusion phenomena for iron and aluminium in the bath. The growth of the interfacial alloy was assumed to be controlled by iron diffusion through this alloy. Tang [10] assumed that the growth of the interfacial alloy layer was controlled by aluminium diffusion in the bath. He proposed a model for nucleation and growth of the alloy layer. Giorgi et al [24] developed a model to predict the concentration profile of iron and aluminium as a function of galvanizing time and the time needed to form a continuous layer. However, the phenomenological models developed so far are inadequate to incorporate all the hysic-chemical processes involved in galvanizing.

\subsection{Artificial Neural Network (ANN)}

In recent times Artificial Neural Network (ANN) is being effectively used in various fields of engineering and science. In the field of multivariate modeling ANN offers many advantages over conventional multiple linear regression methods. Artificial neural networks can identify patterns between the dependent and independent variables in data sets. Furthermore, they can create a specialized regression model or model adjustment for all patterns discovered during analysis.

The true power and advantage of neural networks lie in their ability to represent both linear and non-linear relationships and to learn these relationships directly from the data being modeled. Neural networks deal very effectively with data discontinuities and non-linear transformations. Additionally, neural networks adapt and learn such that they can simulate and choose models correctly for recurring patterns. The dependence of the final coating thickness in galvanizing on input variables is highly nonlinear and coupled in nature. Hence, the ANN modeling approach was chosen in this work. 


\section{Experimental}

\subsection{Experimentation in HDPS}

In HDPS one can change the settings of various inputs under study from one experiment to another. After conducting a matrix experiment, the data from all experiments in the set were taken together and analyzed to determine the effects of different inputs. Zn-coating was made over $200 \mathrm{~mm} \times 120 \mathrm{~mm}$ Cold Rolled sheet samples with thickness of around $0.5 \mathrm{~mm}$ in an HDPS in accordance with the Design of Experiments (DoE). The sheet samples were annealed and dipped in the molten Zinc-bath $(\sim 35 \mathrm{~kg}$ weight $)$.

After galvanizing the coating thickness of all the experimental samples were measured by a magnetic type portable Coating Thickness Gauge (Make: Defalsko, Model: Positector 6000). The thickness of each side of the coated samples was worked out on the basis of 9 measurements covering about $80 \mathrm{~mm} \times 80 \mathrm{~mm}$ area of the central region. The mean of the thickness of both the sides was considered as the coating thickness of one experimental sample. Hence, the coating thickness of each experiment is actually the mean of 18 measurements. Different baths were prepared to vary the Al content of the bath. One experiment was carried out for each set of parameters. DoE was planned such that maximum variations and combination of inputs are possible keeping the number of experiments low. The experiments were conducted in two phases.

\subsection{Sensitivity Analysis}

A sensitivity analysis was carried out to identify the critical variables. Design of experiments was formulated using Taguchi's Orthogonal Array Technique and encompassing the entire range of actual operation of a CGL with 8 process parameters as factors. From Taguchi's Orthogonal Array Selector the corresponding orthogonal array is found to be L18. The ranges and the levels of the 8 factors at which the experiments were carried out in HDPS to generate the data for the sensitivity analysis are presented in Table 1.

Table 1. Factors, Their Ranges and Levels for Sensitivity Analysis

\begin{tabular}{|c|c|c|c|}
\hline S.No. & Factor & Range & Levels \\
\hline 1. & Al-content in bath (\%) [F1s] & $0.12-0.20$ & $0.12,0.20$ \\
\hline 2. & Pb-content $(\%)[\mathbf{F 2}$ s] & $0.015-0.150$ & $0.015,0.095,0.150$ \\
\hline 3. & Bath Temperature ( ${ }^{\circ}$ C) [F3 $]$ & $450-470$ & $450,460,470$ \\
\hline 4. & Residence Time(s) [F4s] & $1.0-3.0$ & $1.0,2.0,3.0$ \\
\hline 5. & Wiping Gas (N2) flow rate (lpm) [F5s] & $200-500$ & $200,350,500$ \\
\hline 6. & Distance between Nozzles (mm) [F6s] & $15-30$ & $15,23,30$ \\
\hline 7. & Wiping Speed (mm/s) [F7s] & $200-500$ & $200,350,500$ \\
\hline 8. & Holding Time before wiping (s) [F8s] & $0.5-1.5$ & $0.5,1.0,1.5$ \\
\hline
\end{tabular}

Out of 8 factors $\mathrm{Pb}$-content in bath \& wiping speed were discarded and the following six dominant factors were identified for inclusion in the development of the predictive model for coating mass/thickness.

Al-content in bath (\%)

[F1]

Bath Temperature $\left({ }^{\circ} \mathrm{C}\right)$

Distance between wiping Nozzles (mm)

Holding Time before wiping (s)

Dipping / Residence Time(s)

Wiping Gas $\left(\mathrm{N}_{2}\right)$ flow rate (lpm)

[F6] 


\section{Development of ANN Model}

\subsection{Training of ANN}

The ranges and the levels of the 6 factors at which the experiments were carried out in HDPS to generate the training data are presented in Table 2.

Table 2. Factors, Their Ranges and Levels for Training the ANN Model

\begin{tabular}{|l|l|l|l|}
\hline $\begin{array}{c}\text { S. } \\
\text { No }\end{array}$ & \multicolumn{1}{|c|}{ Factor } & \multicolumn{1}{c|}{ Range } & \multicolumn{1}{c|}{ Levels } \\
\hline 1. & Al-content in bath (\%) [F1] & $0.10-0.28$ & $0.10,0.12,0.14,0.16,0.18$, \\
& & & $0.20,0.22,0.24,0.26,0.28$ \\
\hline 2. & Bath Temperature $\left({ }^{\circ} \mathrm{C}\right)[\mathbf{F} 2]$ & $445-470$ & $445,450,455,460,465,470$ \\
\hline 3. & Distance between Nozzles (mm) [F3] & $15-17$ & $15,16,17$ \\
\hline 4. & Holding Time before wiping (s) $[\mathbf{F 4}]$ & $0.1-2.1$ & $0.1,1.1,2.1$ \\
\hline 5. & Residence Time(s) [F5] & $1.5-3.5$ & $1.5,2.5,3.5$ \\
\hline 6. & Wiping Gas $\left(\mathrm{N}_{2}\right)$ flow rate (lpm) [F6] & $200-450$ & $200,250,300,350,400,450$ \\
\hline
\end{tabular}

Scaling and de-scaling of input and output data were carried out for efficient network training. In the present work batch-mode training was employed where weights were updated after each epoch for all the samples in the training data set. The error between neural network prediction from the input set used for training and target output was fed back to the neural network to update the internal weights and biases of the neural network. The back propagation training rule was used to adjust the weight and bias vectors of network in order to achieve the desired goal for sum-squared error of the network. The final values for weights and biases are the design values for the network and were used to predict the system performance variable. '

\subsection{The Network Architecture}

A Multi Layer Feed Forward (MLFF) network was selected in the present work. After a large number of trials it was finally decided to have one input layer and three hidden layers and one output layer. Input layer contains six neurons (six factors), $1^{\text {st }}$ hidden layer contains $9,2^{\text {nd }}$ hidden layer contains 6 neurons, $3^{\text {rd }}$ hidden layer contains 3 neurons and output layer contains one neuron (one output). The Network Architectures, called 6-(9-6-3)-1 network, is schematically illustrated in Fig. 1.

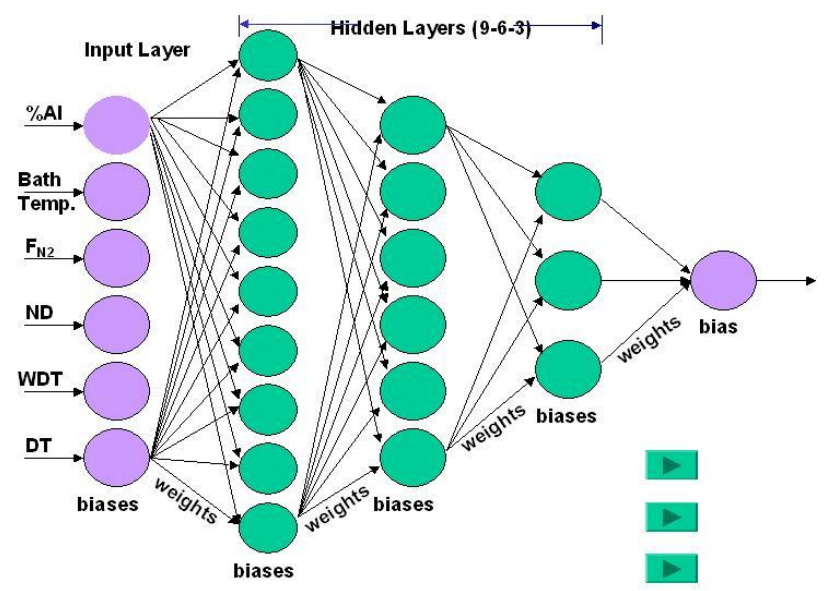

Fig. 1. The network architectures: 6-(9-6-3)-1 network. 
A multiple input-single output relation mapped by an ANN with one hidden layer can be expressed as:

$$
y=F_{2} \cdot\left(w_{2} \cdot F_{1}\left(w_{1} \cdot x+b_{1}\right)+b_{2}\right)
$$

where $x$ is the input vector, $W_{i}$ and $B_{i}$, are connection weight and bias matrices, respectively, y is the output vector, and $F_{i}$ is an activation function, which may be thought of as providing a nonlinear gain for the artificial neuron. Keeping in mind the nonlinear nature of the process, sigmoid functions were chosen for three hidden layers and linear function for final output layer.

$$
F(x)=2 /[1+\exp (-2 x)]-1
$$

The convergence criterion is chosen as mean of sum of the square of the errors for all the experimental findings as 0.018 . The configuration of network described above has $6 \times 8$ no of weights and 8 numbers of biases in the first hidden layer, $8 \times 6$ weights and 6 biases in the second hidden layer and $6 \times 3$ weights and 3 biases in the third hidden layer. For output layer there are $3 \times 1$ numbers of weights and 1 bias. Around 2,000 iterations were required to achieve the desired goal of error level.

The entire operation starting from network architecture, error calculation and training of the network was carried out using Neural Network toolbox of MATLAB. A general purpose MATLAB command line program was developed to use the different functions of the neural network toolbox. The following functions were used.

\subsection{Training Algorithm}

The salient steps are presented below.

Steps of Gradient Based Training Algorithms :

Step 1: Initial guess $\mathrm{k}=1$

Step 2: If $\left(\mathrm{E}_{\operatorname{Tr}}(\mathrm{k})<\mathrm{e}\right.$ (given accuracy criteria) or $\mathrm{k}>\mathrm{kmax}$ (max number of iterations).

Step 3: Calculate $\mathrm{E}_{\operatorname{Tr}}$ and $\frac{\partial E_{T r}}{\partial w}$ using partial or all training Data.

Step 4: Use optimization algorithm to find $\mathrm{w}$.

Step 5: Update weights as $\mathrm{w}=\mathrm{w}+\mathrm{w}$.

Step 6: If all training data are used, then $\mathrm{k}=\mathrm{k}+1$, go to Step 2, else go to Step 3.

Scaled conjugate gradient method was used in the present work.

The expressions for different errors are shown below.

$$
\begin{aligned}
& E_{T r}(w)=\left[\frac{1}{\operatorname{size}(T r) \cdot m} \sum_{k \in T r} \sum_{j=1}^{m}\left|\frac{p_{j}\left(x_{k}, w\right)-t_{j k}}{p_{\max j}-p_{\min j}}\right|^{q}\right]^{1 / q} \\
& E_{T e}(w)=\left[\frac{1}{\operatorname{size}(T e) \cdot m} \sum_{k \in T e} \sum_{j=1}^{m}\left|\frac{p_{j}\left(x_{k}, w\right)-t_{j k}}{p_{\max j}-p_{\min j}}\right|^{q}\right]^{1 / q}
\end{aligned}
$$


$x$ : input of the original modeling problem or the neural network.

$p$ : predicted output of the neural network.

$w$ : internal weights/parameters of the neural network.

$m$ : number of outputs of the model.

$t$ : target data for input set.

$q$ : generally 2 .

\subsection{Data Analysis \& Filtering}

All the experimental data were analyzed \& filtered with respect to the following:

i) The mean \& the maximum of the standard deviation values of the coating thickness

ii) The difference between the mean coating thickness values at the two sides

iii) The effects of individual parameters on the coating thickness

234 data sets (after filtering) were considered for model development.

\subsection{Training \& Validation of Neural Network}

Witten \& Frank [25] and Swingler [17] proposed that one-third of the data be withheld for validation and the remaining two-thirds be used for training and testing. Using this approach to avoid the phenomenon of overtraining, all the 234 experimental data sets were sorted in increasing order of coating thickness. Then half of the data sets were used for training; one-fourth for validation and the remaining one-fourth was marked as test set. Training set, Test set and Validation set followed the order $1,3,5, \ldots ; 2,6,10, \ldots \& 4,8,12, \ldots$ respectively.

\section{Results and Discussion}

The validation set and test set were fed to the network during training session and the network not only calculated the training error but also the error for validation and test set. The network weights and biases were updated based on training error. With continuous updating of weights and biases training error reduced continuously, while the test and validation error might remain constant or increase with updating. At this point the training was stopped though the error of training set does not reach the desired level This methodology is called early stopping of network during training phase. The frequency distribution of the data (Fig. 2) shows that most of the data lie in the range $12-32 \mu \mathrm{m}$.

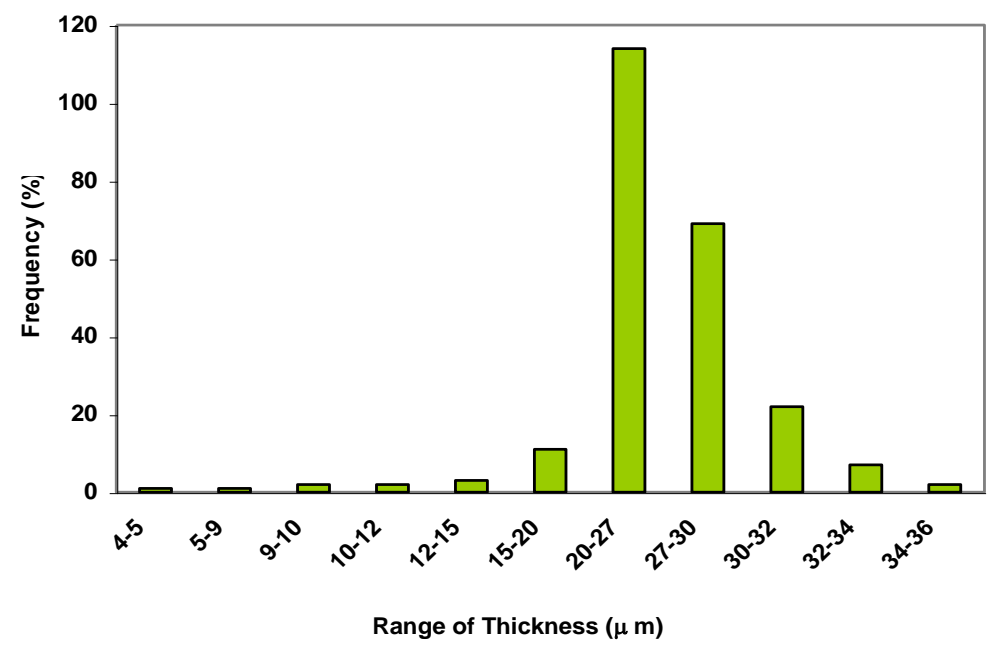

Fig. 2. Frequency distribution of 234 experimental data. 
As ANN model needs a large number of data for proper training and validation, this range with 219 data was selected for final model development. The comparison between model predicted \& actual values and the mean validation error (0.130) are depicted in Fig. 3 and Fig. 4.

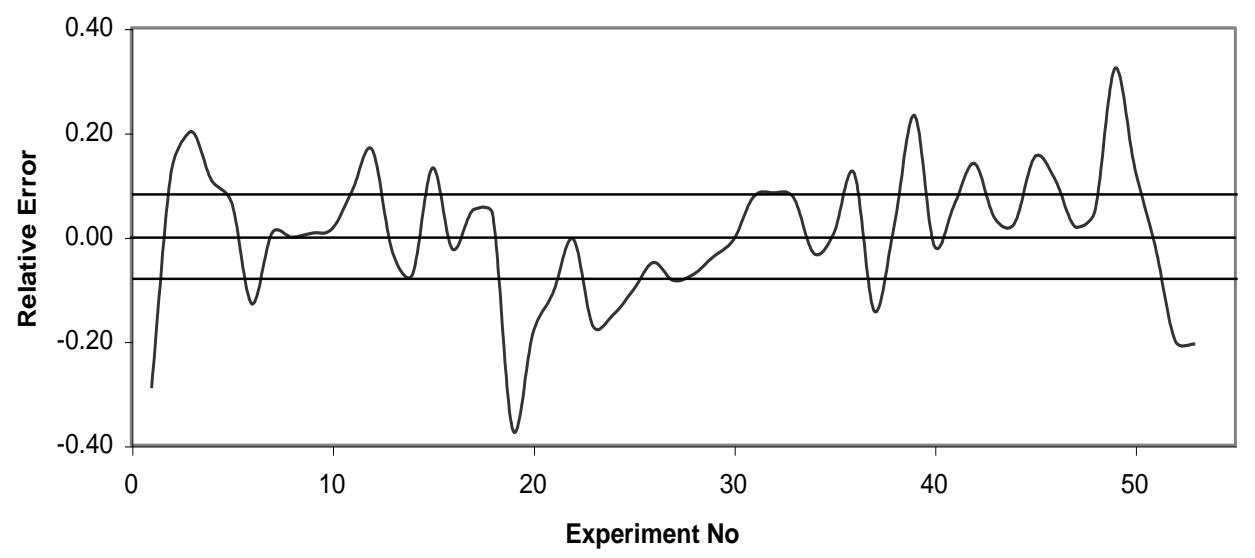

Fig. 3. Relative error between experimental and predicted coating thickness.

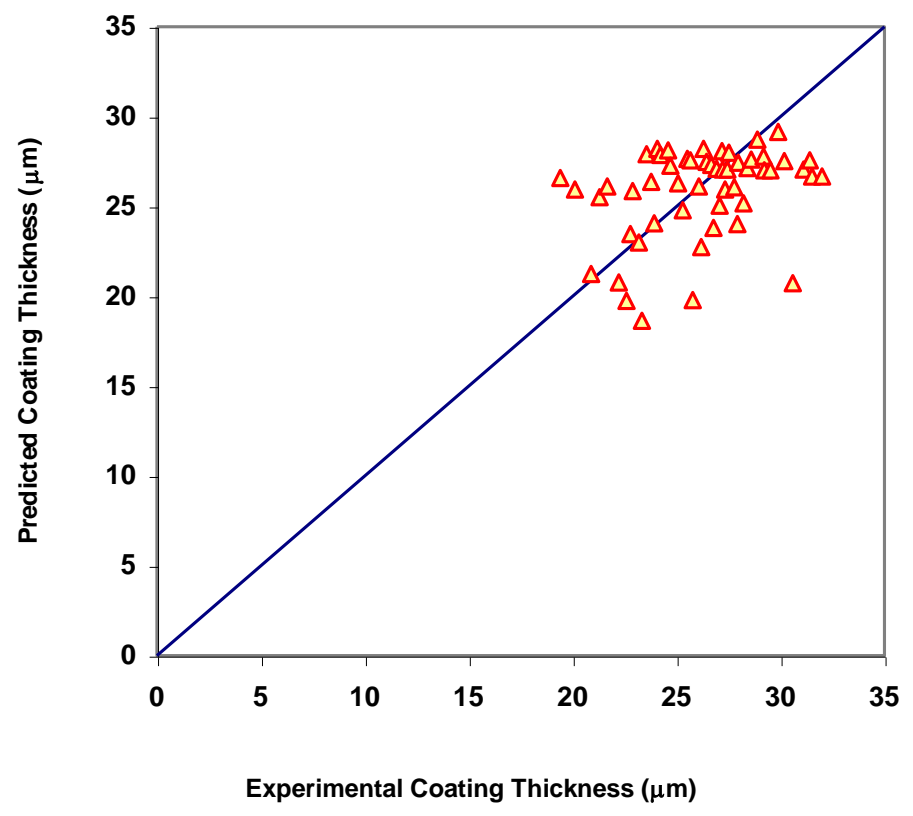

Fig. 4. Comparison between experimental and predicted coating thickness.

The relative errors for model training \& validation are depicted in Table 3.

Table 3. Relative Errors for Model Training \& Validation

\begin{tabular}{|c|c|c|c|c|}
\hline Based on & Range & Data selected / Total Data & Training Error & Validation Error \\
\hline Actual thickness & $12-32$ & $219 / 234$ & 0.110 & 0.130 \\
\hline Maximum thickness & $12-32$ & $219 / 234$ & 0.072 & 0.081 \\
\hline
\end{tabular}

When the error was calculated on the basis of the maximum thickness, which is done quite often to avoid large 
error arising out at lower values, the mean training \& validation errors decreased to 0.072 and 0.081 respectively (Table 3).

\section{Conclusions}

The following conclusions may be made from the outcome of this work:

1) An ANN model has been developed to predict the coating thickness in a hot dip galvanizing process. Multilayered feed-forward network with a 6-(9-6-3)-1 architecture is found suitable for the model. The ANN model developed is found to be effective in predicting the galvanizing coating thickness.

2) As the thickness data was scattered over a wide range and there was a reasonable concentration of data in the range of $12-32 \mu \mathrm{m}$, such data range was selected for model training and validation. The relative errors of the model for the thickness range of $12-32 \mu \mathrm{m}$ with a reasonable concentration of data in training and validation were 0.110 and 0.130 respectively based on actual thickness and were 0.072 and 0.081 respectively based on maximum thickness.

\section{References}

[1] Butler, J. J., Beam, D. J., \& Hawkins, J. C. (1970). The development of air coating control for continuous strip galvanizing. Iron \& Steel Engineer, 47(2), 77- 86.

[2] Oren, E. C., Ptashnik, W. S., \& Martin, D.C. Galvatech'95 Conference Proceedings (pp. 67-73).

[3] Davene, L. Dondin, J.K. Kim \& S.J. Kim. Galvatech'95 Conference Proceedings (pp. 205-213).

[4] Dubois, M., Riethmuller, M. L., Buchlin, J. M., \& Arnalsteen, M. Galvatech'95 Conference Proceedings (pp. 667673)

[5] Caignard, S., \& Dubois, M. Gatvatech'04 Conference Proceedings (pp. 207-216).

[6] Deote, A. P., Gupta, M. M., Zanwar, D. R. (2012). Process parameter optimization for zinc coating weight control in continuous galvanizing line. International Journal of Scientific \& Engineering Research, 3(11), 1-6.

[7] Liberski, P., Tatarek, A., \& Mendala, J. (2013). Investigation of the initial stage of hot dip zinc coatings on iron alloys with various silicon contents. Solid State Phenomena., 212, 121-126.

[8] Blohm, H. Intergalva'79 (pp. 215-218). London: Zinc Development Association.

[9] Townsend, C. S., \& Bilski, W. C. (1988). Closed-loop control of coating weight on a hot dip galvanizing line. Iron \& Steel Engineer, 65(7), 44-47.

[10] Tang, N. Y. (1995). Modeling Al enrichment in galvanized coatings. Metallurgical and Materials Trans. A, 26(7), 1699-1704.

[11] Chakkingal, U., \& Wright, R. N., Galvatech'95 Conference Proceedings (pp. 823-829).

[12] Laviosa, V., Milani, A., \& Goodwin, F. E. (1998). Zinc-based steel coating systems: Production \& performance. In F. E. Goodwin(Ed.), The Minerals Metals \& Materials Society (pp. 83-91).

[13] Toussaint, P., Segers, L., Winand, R., \& Dubois, M. (1998). Mathematical modelling of Al take-up during the interfacial inhibiting layer formation in continuous galvanizing. ISIJ International, 38(9), 985-990.

[14] Rios, P. R., Galvatech'01 Conference Proceedings (pp. 519-525).

[15] Migliorino, D., Cervellini, G., \& Etcheverry, J. I., Galvatech'04 Conference Proceedings (pp. 893-903).

[16] Markward, S. W. \& Lu, Y. Z. (1995). Integrated neural system for coating weight control of a hot dip galvanizing line. Iron \& Steel Engineer, 72(11), 45-49.

[17] Swingler, K. (1996). Applying Neural Networks: A Practical Guide. San Francisco: Morgan Kaufman.

[18] Mousavifard, S. M., Attar, M. M., Ghanbari, A., \& Dadgar, M. (2015). Application of artificial neural network 
and adaptive neuro-fuzzy inference system to investigate corrosion rate of zirconium-based nano-ceramic layer on galvanized steel in 3.5\% $\mathrm{NaCl}$ solution. Journal of Alloys and Compounds, 639, 315-324.

[19] Wang, X. A., \& Mahajan, R. L. (1996). Artificial neural network model-based run-to-run process controller, IEEE Trans. On Components, Packaging \& Manufacturing Technology: Part C, 19(1), 19-26.

[20] Tarassenko, L. (1998). A Guide to Neural Computing Application. UK: Arnold Publications.

[21] Rendueles, S. L., Gonzalez, J. A., Ortega, F., \& Vergara, E. Galvatech'01, Conference Proceedings (pp. 540-547).

[22] Srinivasan, V. S., Shankar, P., \& Raj, B. (2004). Citation report of Dr. Baldev Raj. IIM Metal News, 7(3), 19-23.

[23] Maitra, S., Pal, A. J., Bandopadhyay, N., Basumazumdar, A., \& Das, A. K. (2007) Use of artificial neural network for modelling the interaction between fly ash and lime under steam curing. Trans. Of Indian Ceramic Soc., 66(4), 187-192.

[24] Giorgi, M. L., \& Guillot, J. B. (2004). Modeling of the Kinetics of Galvanizing Reactions. Galvatech '04 Conference Proceedings (pp. 703-711).

[25] Witten, I. H., \& Frank, E. (2000). Data Mining: Practical Machine Learning Tools and Techniques with Java Implementations. San Francisco: Morgan Kaufmann.

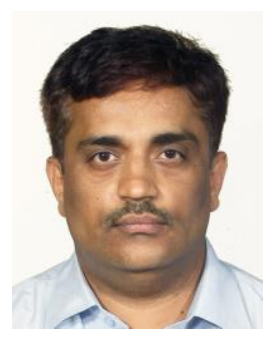

S. K. Shukla received B. Tech and M. Tech degree in Materials \& Metallurgical Engg. from Indian Institute of Technology, Kanpur, India in 1993 and 1996 respectively.

Presently, he is working as Assistant General Manager in R\&D Centre of Steel Authority of India Ltd. (SAIL), Ranchi, India. Since joining this centre in 1996, he is involved in development of various special steel products for sail steel plants such as extra deep drawing steel, line pipe steel, microalloyed steel for cold drawn pipes and coated products. He has published more than 20 papers, filed 3 patents and 2 copyrights. He is a life member of Indian Institute of Metals (IIM). 